\title{
EDITORIAL
}

\section{CARE OF DEMENTIA IN CANADA: A COLLABORATIVE CARE APPROACH WITH A CENTRAL ROLE FOR THE PRIMARY CARE PHYSICIAN}

\author{
F. MASSOUD 1 , P. LYSY², H. BERGMAN ${ }^{3}$
}

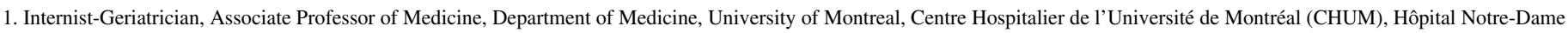

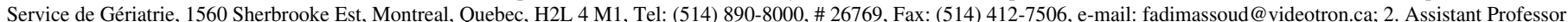
of Family Medicine, McGill University; 3. The Dr. Joseph Kaufmann Professor of Geriatric Medicine, Professor of Medicine, Family Medicine and Oncology, McGill University and Jewish General Hospital, Vice-président et directeur scientifique, Fonds de la recherche en santé du Québec
\end{abstract}

A chronic and complex disease such as dementia requires organized and integrated care from all the actors involved. Management of dementia entails ensuring early detection and diagnosis, starting and maintaining the appropriate pharmacological treatment, and most importantly providing ongoing support to the patient and his caregivers as they go through the different stages of the disease, many of which impose difficult decisions and dispositions (medico-legal issues, driving, long-term care, etc.). This makes it impossible to handle by a single healthcare professional and necessitates a team effort. Most, but unfortunately not all, patients with dementia in Canada have a primary care physician (PCP) who will provide medical care and coordinate referrals to specialists, other professionals, and community services. Hence, these physicians usually represent the patient's first contact with the health system and the first level in the diagnostic process of a cognitive disorder. Since 1989, three Canadian Consensus Conferences on the Diagnosis and Treatment of Dementia (CCCDTD) have repeatedly recommended that PCP's be proficient in the diagnosis and treatment of uncomplicated dementia $(1,2)$. These recommendations give pragmatic and evidence-based advise on diagnosis, work-up (including neuroimaging and other biomarkers), and treatment of different types of dementia (3). The most recent CCCDTD was held in Montreal in 2006, and one of its recommendations states: "Most patients with dementia can be assessed and managed adequately by their primary care physicians" (3). Its findings were widely disseminated and a set of small group based educational modules aimed at PCPs will be launched nationally this October. Recent data on drug utilization of cholinesterase inhibitors in Quebec show that indeed most prescriptions are being written by PCP's (4). It is probable that some of these prescriptions are written upon recommendation after evaluation by a specialist, but it is safe to assume that most of these medications are being started (and renewed) by a PCP. In fact, the study shows that between the years 2000 and 2003, the percentage of index prescriptions by a PCP rose from $67 \%$ to $78 \%$, suggesting gained expertise and comfort with the use of this class of medication (5). Presently, PCP's are expected to make the diagnosis of dementia in most uncomplicated cases and to initiate and manage pharmacological treatment when indicated. They also coordinate referral to a specialist or a Received August 28, 2009

Accepted for publication September 27, 2009 specialized clinic if available. In many Canadian provinces, community based health and social service centers are also available to assist the PCP in his management of dementia patients. They may offer services to support functional deterioration, respite care for caregivers, and coordinate longterm placement.

Despite their key role, many PCP's still feel uncomfortable with the management of dementia. This situation is complicated by the limited access to work-up facilities (including neuroimaging) or referral settings in certain areas. Primary Care Physicians in most Canadian provinces are not adequately remunerated for their work in the diagnosis and management of dementia. Their fees are not adapted to the sometimes lengthy diagnostic process. Reimbursement requirements for pharmacological treatments in all provinces add to paperwork and time required for adequate follow-up of patients. These factors lead to unnecessary delays in diagnosis and treatment of patients with dementia and undue referrals to specialized centers such as Memory Disorders Clinics which, despite their mission of evaluating atypical and complicated dementia cases, often have a significant load of relatively uncomplicated patients. This in turn perpetuates the cycle of limited access to specialized evaluations. Six to 12 months waiting lists are not uncommon.

Certain provinces in Canada are taking initiatives to deal with these challenges. For example, a Quebec government task force chaired by Howard Bergman recently presented to the Minister of Health their report proposing pragmatic solutions. This recently published document entitled "Meeting the Challenge of Alzheimer's disease and Related Disorders. A Vision Focused on the Individual, Humanism, and Excellence. Report of the Committee of Experts for the Development of an Action Plan on Alzheimer 's disease and Related Disorders" (6) underscores the important role of PCP's in dementia care. It is based on the chronic disease model as well as the collaborative care model (7) and recommends a close partnership between the PCP, a nurse clinician trained in the care of dementia, the patient and his caregivers. This approach would be integrated within primary care and the existing system. The Bergman report proposes uniting government, university and private sector expertise and resources in a massive education and training campaign for primary care physicians and nurses, in 


\section{A COLLABORATIVE CARE APPROACH WITH A CENTRAL ROLE FOR THE PRIMARY CARE PHYSICIAN}

particular. The report also proposes specific and adapted remuneration for primary care physicians following patients with Alzheimer's disease. To facilitate this role, clinical practice guidelines, standard protocols and instruments will be developed for the identification, monitoring and treatment of dementia and its complications, allowing for management of most cases, and for prioritized referral and access to specialized clinics. Two levels of specialized clinics are proposed. Secondary care local and regional Memory Disorders Clinics with rapid access for the PCP will permit the referral of challenging cases in terms of diagnosis or treatment, and would include physicians specialized in the management of dementia and its complications (geriatrician, neurologist, geriatric psychiatrist, or specialized family physician). Tertiary Memory Disorders Clinics affiliated with academic institutions, offering integrated care by one or several specialists as well as other health-care professionals (specialized nurse, neuropsychologist, ocuupational therapist, etc.). The main characteristics of these clinics are a high level of expertise in the care of most complicated cases of dementia, involvement in research and training, as well as technology evaluation. This close partnership between primary care and specialized care is essential for the success of such an initiative. Other resources are proposed to enhance this model including dementia support centers (under the leadership of the Alzheimer Society), teams specialized in the management of behavioural complications of dementia, and psychosocial services. A randomized controlled trial of a similar collaborative care model for AD in the US showed improvement in quality of care and in the management of behavioural complications of dementia. It also led to a significant reduction of caregiver burden at 12 and 18 months (7)

Care of dementia in Canada faces several challenges that will need to be addressed over the next few years to meet with the growing demand. Collaborative care models with a central and coordinating role for the PCP are proposed to meet with these challenges as patients increase in numbers and expectations.

\section{References}

1. Patterson CJ, Gauthier S, Bergman H, Cohen CA, Feightner JW, Feldman H, et al. The recognition and assessment and management of dementing disorders conclusions from the Canadian Consensus Conference on Dementia. CMAJ 1999; 160 (suppl): S1-15

2. Chertkow H. Introduction: the third Canadian Consensus Conference on the Diagnosis and Treatment of Dementia 2006. Alzheimer's \& Dementia 2007;3:262265 .

3. Hogan DB, Bailey P, Carswell A, Clarke B, Cohen C, Forbes D, et al. Management of mild to moderate Alzheimer's disease and dementia. Alzheimer's \& Dementia 2007; 3:355-384

4. Massoud F, Dorais M, Charbonneau C, Lescrauwaet, Boucher JM, LeLorier J. Drug utilization review of cholinesterase inhibitors in Quebec. Can J Neurol Sci 2008; 35 :508-509

5. Massoud F, Dorais M, Charbonneau C, Lescrauwaet, Boucher JM, LeLorier J. Drug utilization review of cholinesterase inhibitors in the province of Quebec, Canada. Neurobiology of Aging 2004; 25 (S2) : S221

6. Committee of Experts for the Development of an Action Plan on Alzheimer's disease and Related Disorders. Meeting the Challenge of Alzheimer's disease and Related Disorders. A Vision Focused on the Individual, Humanism, and Excellence. http://www.rqrv.com/fr/document/rapport alzheimer.pdf. Comité d'Experts en Vue de l'Élaboration d'Un Plan d'Action Pour la Maladie d'Alzheimer. Relever de défi de la maladie d'Alzheimer et de maladies apparentées :une vision centrée sur la personne, l'humanisme et l'excellence. http://www.rqrv.com/fr/document /rapport_alzheimer.pdf

7. Callahan C, Boustani MA, Unverzagt FW, Austrom MG, Damush TM, Perkins AJ et al. Effectiveness of collaborative care for older adults with Alzheimer disease in primary care. A randomized controlled trial. JAMA 2006;295:2148-2157. 